CAHIERS DE

NARRATOLOGIE

\section{Cahiers de Narratologie}

Analyse et théorie narratives

$36 \mid 2019$

Rhétorique et représentations de la culture mafieuse. Images, rituels, mythes et symboles

\title{
Ré-visions de la trilogie du Parrain : anatomie d'un mythe à l'époque des séries télévisées
}

\section{Andrea Meccia}

Translator. Manuela Bertone (de l'italien)

\section{(2) OpenEdition}

\section{Journals}

Electronic version

URL: http://journals.openedition.org/narratologie/9776

ISSN: 1765-307X

Publisher

LIRCES

Electronic reference

Andrea Meccia, «Ré-visions de la trilogie du Parrain : anatomie d'un mythe à l'époque des séries

télévisées », Cahiers de Narratologie [Online], 36 | 2019, Online since 20 December 2019, connection on 20 December 2019. URL : http://journals.openedition.org/narratologie/9776

This text was automatically generated on 20 December 2019.

Article L.111-1 du Code de la propriété intellectuelle. 


\title{
Ré-visions de la trilogie du Parrain : anatomie d'un mythe à l'époque des séries télévisées
}

\author{
Andrea Meccia
}

Translation : Manuela Bertone (de l'italien)

Le cinéma est l'arme la plus puissante de l'ère moderne. Et c'est d'autant plus vrai si l'on considère

qu'il est destiné à changer radicalement dans les années à venir. Il est destiné à devenir électronique, numérique, et à créer les rêves et les hallucinations de

l'avenir.

Francis Ford Coppola, 1979

\section{Réunion de famille}

1 En avril 2017, quarante-cinq ans après la sortie en salles du Parrain [The Godfather] de Francis Ford Coppola - l'œuvre qui, en trois volets (1972, 1974, 1990), raconte l'épopée criminelle de la famille Corleone - le Tribeca Film Festival a célébré les personnages de la dynastie sicilo-américaine en les rassemblant pour une soirée de gala ${ }^{1}$ et un binge watching $^{2} \mathrm{du}$ film. Le lieu choisi pour l'occasion est le Radio City Music Hall, déjà exploité lors du tournage pour mettre en scène l'histoire d'amour entre deux protagonistes du film, Michael Corleone (Al Pacino) et Kay Adams (Diane Keaton). 377 minutes de visionnage : telle est la durée de la projection des deux premières parties de la plus célèbre saga mafieuse de l'histoire du cinéma.

2 Né de la plume de l'écrivain italo-américain Mario Puzo, le roman The Godfather, publié en 1969 par la maison d'édition new-yorkaise G.P. Putnam's Sons, s'est vendu à 9 millions d'exemplaires en deux ans. L'idée d'écrire une œuvre destinée à "s'imposer comme modèle parfait du best-seller mondial, transformé au fil du temps en une pépinière de divertissement multimédia et même en label idéal pour les offices du 
tourisme $»^{3}$, naît en 1966, dans le sillage des confessions de Joe Valachi, le gangster «traître " qui, en 1964, devant la commission d'enquête présidée par le sénateur McClellan, avait utilisé pour la première fois l'expression «la cosa nostra» pour «révéler [...] le nom, utilisé par les initiés eux-mêmes, d'une société secrète dans laquelle on entrait suite à un rituel et en prêtant serment $»^{4}$. Quelques années plus tard, en 1971, Gay Talese, le pionnier du new journalism, suite à la sanglante Banana War, publie Honor Thy Father [Ton père honoreras] une sorte de biographie autorisée des Bonanno, une des cinq familles de la mafia new-yorkaise. Talese, lui aussi d'origine italienne, décrit Bill, le fils aîné de Joseph Bonanno, aux prises avec le roman de Puzo :

Il avait passé la moitié de la nuit à lire le livre qu'il tenait à la main, Le Parrain, roman sur la mafia, qui lui plaisait beaucoup. Arrivé à peu près à la moitié, il était certain que l'auteur, Mario Puzo, était fort bien renseigné sur cette société secrète. Le personnage principal du roman, Don Vito Corleone, lui paraissait vraisemblable. Il se demandait si son nom ne dérivait pas en partie de «Don Vito " Genovese. Quant à l'agglomération de Corleone, elle existe et se trouve à quelque distance de la côté, en Sicile occidentale, au sud-est de Castellammare. Bill trouvait que son propre père possédait bien des qualités attribuées par l'écrivain à Vito Corleone, surtout le calme et la subtilité. Mais d'autres traits de caractère lui rappelaient feu Thomas Lucchese. Comme Don Vito Corleone, Lucchese avait des amis influents parmi les dirigeants du Parti démocrate à New York pendant les années 1950, des gens qui, paraît-il, rendaient des services exceptionnels à ceux qui finançaient généreusement leur campagne électorale. [...] Les Siciliens décrits dans Le Parrain non seulement Don Vito Corleone et son fils Michael, qui était passé par l'université et avec qui Bill s'identifiait, mais aussi d'autres personnages - étaient doués d'un courage et d'un sens de l'honneur impressionnant, caractéristique qui n'avait pas résisté à l'érosion des temps nouveaux. L'action du roman se déroulait peu après la Seconde Guerre mondiale ; peut-être la mafia était-elle alors telle que la peignait l'auteur. Tout en continuant de lire, Bill regretta une période qu'il n'avait d'ailleurs pas connue personnellement ${ }^{5}$.

3 Quelques mois plus tard, en 1972 (l'année de début de l'affaire Watergate), The Godfather, grande fresque d'une Amérique se dirigeant vers les tranchées imaginaires de la guerre froide, vit la première d'une longue série de média-morphoses : transformé en long-métrage par Paramount ${ }^{6}$, il est répertorié comme œuvre cinématographique appartenant au genre «drama», au sous-genre "gangster, with songs » ${ }^{7}$. D'après Jack Shadoian Le Parrain constitue

une épopée domestique [...] sur les conséquences du capitalisme américain dans la vie familiale. La colère morale des films des années 1950 consacrés à la mafia et au crime organisé n'est plus d'actualité [...]. Les gangsters et les non gangsters sont les uns comme les autres paralysés dans le vide laissé par l'impossibilité du rêve américain dans une société post-Vietnam, post-Watergate [...]. Michael est un monstre, certes, mais nous comprenons et acceptons les raisons de sa monstruosité. [...] Les spectateurs comprennent et regrettent l'inéluctabilité de sa situation, car leurs propres vies se sont également endurcies après la disparition des rêves de la fin des années soixante 8 .

Du point de vue commercial, Le Parrain est une « opération [...] parfaitement adaptée à la vague des nouvelles grandes productions » de l'industrie culturelle des Etats-Unis : bien plus qu'un simple film, c'est "une holding, une entreprise planétaire, une opération bancaire, un séisme financier $»^{9}$. Du point de vue de la distribution, comme le rappelle Gian Piero Brunetta, on inaugure avec Le Parrain « cette politique de saturation [saturation selling] qui, pour les films les plus coûteux, consiste à augmenter vertigineusement le nombre de copies distribuées [...] de manière à récupérer 
rapidement les capitaux investis $»^{10}$. Les chiffres sont stupéfiants. Pendant les vingtcinq premiers jours de programmation, le film rapporte 26 millions de dollars. Fin 1972, il atteint le record de 43 millions. En une année d'exploitation, il atteint les 81 millions. «Paramount dépensait environ un million de dollars par semaine en publicité et promotion, et gravait [...] un grand nombre de copies [...] après l'attribution de l'Oscar à Coppola, ils en ajoutèrent plus de $1000 »^{11}$.

5 La trajectoire artistique et commerciale du Parrain s'inscrit dans le contexte du renouveau de New Hollywood et d'une société aux prises avec les horreurs de la guerre du Vietnam. Inaugurant « le gangster movie du cinéma moderne ", Le Parrain se prépare «à occuper une place d'honneur dans la culture de masse et dans la mémoire collective des générations à venir $»^{12}$. Dans ses images grandiloquentes et mythopoïétiques se concentre sa capacité à saisir l'esprit du temps, à offrir à l'industrie des médias de nouveaux schémas narratifs, à (ré)inventer l'univers communicatif (verbal et non verbal) de la mafia sans même que le mot «mafia» ou l'expression «Cosa Nostra» ne soient utilisés dans le scénario, où en revanche, le registre de l'apologie est sollicité à plusieurs reprises ${ }^{13}$.

\section{Les filleuls de « Don Francis »}

Dès 1972, "profitant de la campagne publicitaire mise en place autour du film sur le "Parrain" [...] pour utiliser une tendance manifestement rentable ${ }^{14}$, les épigones de Coppola commencent à investir les grands écrans italiens. De Hong Kong, pas moins de trois longs-métrages consacrés à la criminalité organisée : Il padrino di Hong Kong (Tieng Peng, 1973 ; titre original : The Godfather of Hong Kong), en 1977 Il padrino cinese e gli ultimi giorni di Bruce Lee (Lai Chien, 1974; titre original: Chinese Godfather) et Il padrino di Chinatown (Chang Cheh, 1977).

7 Don Vito Corleone en personne refait son apparition grâce à Nardo Bonomi, auteur de La mano lunga del Padrino et à Frank Agrama, réalisateur de L'amico del Padrino, " un produit dérivé de mauvaise qualité $\aleph^{15}$ qui surfe sur la vague de Puzo. En 1973, s'inspirant du premier chapitre de la saga, Vittorio Schiraldi réalise le policier Baciamo le mani. Mariano Laurenti, réalisateur prolifique de films musicaux, de comédies et de comédies sexy, tourne la parodie Il figlioccio del Padrino [le filleul du Parrain], inspiré du premier épisode de la trilogie, sans toutefois en respecter l'intrigue et les rôles. Il s'agit en effet de la reprise du voyage de Michael Corleone en Sicile, dans le village natal de son père. Cette fois-ci, le personnage s'appelle Oronzo Musumeci (interprété par l'acteur comique Franco Franchi), il est le filleul du boss mafieux italo-américain Don Vincenzo Russo (Maurizio Arena à l'écran), accueilli en Sicile par un autre mafieux, Don Salvatore Trizzino (Saro Urzì) ${ }^{16}$, père de la jeune Apollonia, qui a déjà perdu quatre maris morts assassinés et qui épousera Oronzo. Quant au toponyme "Corleone », il semble s'imposer également comme label spatial exploitable dès le titre : Corleone (1978) est en effet un film de Pasquale Squitieri se déroulant dans la Sicile des luttes syndicales paysannes des années 50, et Da Corleone a Brooklyn (1979) un policier italo-américain d'Umberto Lenzi. Au fil des décennies, Corleone devient une étape obligée du tourisme cinématographique. Les scènes siciliennes du Parrain et du Parrain II, tournées par Coppola dans la Sicile orientale, à Savoca et Forza d'Agrò, à quelques kilomètres de Taormina, on fait de ces villages des sites incontournables pour les touristes visitant l'île. Coppola a reconstruit « un monde pas si différent du monde primitif dont est issue 
la famille de la fiction. Et il n'y avait pas de différence entre le monde sicilien cruel où Vito était né et celui que Michael connaît [...] des années plus tard $»^{17}$. Le fait d'avoir accueilli l'équipe du tournage et d'incarner une sicile prétendument éternelle et immuable, est désormais partie intégrante de l'identité du village de Savoca. On a érigé un monument représentant Coppola derrière une caméra à quelques mètres du bar Vitelli, où Michael rencontre le père d'Apollonia ${ }^{18}$. Comme l'a souligné Fred Gardaphé, "dans Le Parrain, la Sicile devient pour Don Corleone ce que Krypton était pour Superman, un lieu d'origine légendaire : celui qui en fait l'expérience est élevé au rang de héros, sinon de dieu ${ }^{19}$.

Par ailleurs, il est intéressant de rappeler que grâce à ce long-métrage, placé par l'American Film Institute au deuxième rang du classement des meilleurs films américains de l'histoire du cinéma ${ }^{20}$, un curieux réseau en celluloïd se crée, fait de voix et de visages qui se croisent entre l'Italie et les Etats-Unis. Ainsi, par exemple, Corrado Gaipa, l'acteur qui a prêté sa voix au Prince de Salina en doublant Burt Lancaster dans Le Guépard [Il Gattopardo] de Luchino Visconti (1963) et à Don Mariano Arena, interprété par Lee J. Cobb dans La Mafia fait la loi [Il giorno della civetta] de Damiano Damiani (1968), joue avec Coppola dans le rôle de Don Tommasino. La même année, il est le protagoniste de Le boss [Il boss] de Fernando Di Leo, avec Richard Conte, qui a joué le rôle de Don Barzini dans Le Parrain. Conte devient l'incontournable du cinéma policier italien : il est à nouveau à l'écran dans Salut les pourris [Il poliziotto è marcio] également de Di Leo (1974), Tony Arzenta de Duccio Tessari (1973), Anastasia mio fratello... de Steno (1973), Rue de la violence [Milano trema - La polizia vuole giustizia] de Sergio Martino (1973). Gastone Moschin, protagoniste culte du film noir Milan calibre 9 [Milano calibro 9] de Di Leo (1972), sera Don Fanucci dans Le Parrain II. Parmi les gardes du corps de Michael Corleone en Sicile on retrouve Franco Citti, l'acteur fétiche de Pier Paolo Pasolini, et Angelo Infanti, qui la même année sera Lucky Luciano dans Cosa Nostra [Joe Valachi - I segreti di Cosa Nostra] de Terence Young, précédant de peu Gian Maria Volonté, l'icône du cinéma politique italien, qui jouera le rôle-titre dans Lucky Luciano de Francesco Rosi (1973).

Voici comment Rosi explique son choix :

Coppola avait présenté l'image de la mafia. Il était entré dans ses comportements les plus authentiques, ceux de la famille : une nouveauté. Même si je dois dire que l'idée de la montrer de l'intérieur, de cette façon, semble traduire un regard un peu léger sur les crimes et les aberrations de la pègre. Alors moi, je me suis dit que j'allais raconter le vrai parrain né en Italie et ensuite parti au-delà de l'océan. [...] J'en ai parlé à Cristaldi [Franco Cristaldi, le producteur, $n d t$ ]. Et Franco espérait certainement profiter du succès du Parrain ${ }^{21}$.

10 Il est tout aussi important de mentionner L'altra faccia del Padrino de Franco Prosperi (1973) et La grande bouffe [La grande abbuffata] de Marco Ferreri (1973). Dans le film de Prosperi, c'est à Alighiero Noschese, imitateur versatile et talentueux, qu'est confié le rôle du boss Don Vito Monreale (dont le nom est un toponyme sicilien). Avec son " visage construit, malléable, docile au maquillage, au coiffage, capable de disparaître en tant qu'homme et de ressusciter en tant que masque $»^{22}$, Noschese livre l'une de ses meilleures interprétations, imitant les mouvements et les gestes de Don Vito Corleone ainsi que la voix (italienne) que Giuseppe Rinaldi avait prêtée à Marlon Brando.

11 Même dans le film de Ferreri, qui met en scène des adieux à la vie déchirants et grotesques, interprétés par Marcello Mastroianni, Philippe Noiret, Michel Piccoli et Ugo Tognazzi, une petite place est faite à une citation du Parrain. C'est Tognazzi, dans l'un 
de ses grands rôles satiriques, qui parodie Brando-Corleone, se déguisant en Parrain lors de ce dernier dîner raffiné et hyperbolique.

Du Parrain se dégage somme toute une force narrative tragique semblable à celle du Macbeth shakespearien : « Macbeth et Le Parrain entraînent le spectateur dans le monde du péché : les protagonistes évoluent dans la guerre, dans les vapeurs du sang; la trahison mine les racines de la confiance, de l'amitié et de l'amour; dans les deux ouvrages, le sujet principal est le péché accablant des meurtres que l'on a commis ${ }^{23}$.

Le style classique, grandiloquent, fait de lenteurs et de rebondissements, dont Coppola se sert pour raconter l'épopée de la famille Corleone, captive et colonise l'imaginaire collectif et crée un réseau de symboles, d'icônes, tant à l'intérieur qu'à l'extérieur de l'univers mafieux, comme dans un inextricable jeu de miroirs.

\section{Connaissez-vous Corleone?}

14 Aborder l'histoire de la mafia italienne de la deuxième moitié du XXe siècle, c'est aussi être accompagné par le toponyme Corleone, par l'adjectif «corléonais», par la parabole criminelle de ceux que la chronique et l'histoire ont appelés « les golpistes de Cosa Nostra», des truands tels que Luciano Liggio $^{24}$, Salvatore Riina, Bernardo Provenzano, tous nés à Corleone, village perché situé dans la zone géographique de Palerme. Ils sont partis de ce village, dans l'après-guerre, pour aller à la conquête de Palerme, la capitale sicilienne où, comme l'a écrit Giuseppe Fava, «la mort [était] différente. Plus profonde, plus mystérieuse et fatale $»^{25}$.

15 A partir des années 50 et avec une virulence particulière entre les années 70 et le début des années 90, les médias italiens seront tristement envahis par les prouesses de la faction qu'ils représentent, la "Nazimafia», qui a longuement bercé le projet totalitaire de «faire coïncider l'État [...] avec Cosa Nostra et Cosa Nostra avec la société ${ }^{26}$. À la manière des terroristes, ils avaient pour objectif d'éliminer en série des serviteurs de l'État, des pans entiers de la société civile, des familles rivales. L'un des premiers reportages de la Rai (la télévision publique) sur la mafia, réalisé en 1962 par Gianni Bisiach, avait justement pour titre Rapporto da Corleone [compte rendu depuis Corleone $]^{27}$.

16 Comme l'a noté l'historien anglais John Dickie, «lorsque l'auteur du Parrain, Mario Puzo choisit un lieu de naissance pour son héros, Don Vito Corleone (né Andolini), il tomba par un pur hasard sur la ville qui a donné au monde les hommes d'honneur les plus redoutés et puissants de Cosa Nostra ${ }^{28}$. Et, au sujet de Liggio/Leggio, arrêté en 1974 à Milan, il a précisé :

Les photos les plus connues de Luciano Leggio datent de sa comparution devant le tribunal de Palerme en 1974. En les voyant, on ne peut s'empêcher de penser qu'il avait résolu pour l'occasion d'imiter Marlon Brando en Don Corleone. Avec son cigare, la lourde mâchoire et son air arrogant, la ressemblance est assez bonne. Mais le visage de Leggio était déjà tristement célèbre bien avant la sortie du Parrain. Le rapport de la Commission antimafia sur Leggio, publié l'année même de la sortie du film, n'est pas un document à se soucier de frivoles apparences. Il témoigne pourtant de la fascination des juges pour «sa grosse figure ronde et froide», pour son air « de mépris ironique ». Si le parrain du film était tel que la Mafia aime à se voir - plein de sagesse et soucieux avant tout de sa famille - les traits de Luciano Leggio évoquaient en revanche l'instabilité et la terreur. Si les lourdes paupières de 
Brando donnaient à son personnage une sorte de noble réserve, les yeux fixes de

Leggio laissaient penser qu'il était aussi versatile que malveillant ${ }^{29}$.

Auprès de l'opinion publique, le sens du mot "Corleone " est brièvement resté en suspens, pouvant indiquer un nom de famille d'origine littéraire et un lieu réellement existant; puis il est rapidement devenu une «métaphore des racines siciliennes de la mafia italo-américaine », un sombre "mélange de terreur et de sens chaleureux de la famille $\Perp^{30}$. Pendant ce temps, Le Parrain s'installe fermement dans le code culturel mafieux, au point de constituer un élément de l'identité des clans, des boss et de leurs affiliés.

\section{Les Casamonica et les Greco}

En inversant l'ordre chronologique, prenons deux exemples où réalité et fiction sont incroyablement imbriquées : d'abord les Casamonica, famille d'origine tsigane installée depuis des décennies à Rome, définie « association de malfaiteurs de type mafieux » par la Cour de Cassation italienne en 2019; ensuite les Greco, famille de l'aristocratie criminelle au passé pluriséculaire, ayant prospéré à l'ombre des plantations d'agrumes de la Conca d'Oro palermitaine.

Rome, août 2015, quartier Cinecittà, secteur sud-est de la ville. Une scène de foule est tournée dans la basilique Saint-Jean Bosco, un lieu sacré déjà immortalisé par Federico Fellini dans La Dolce Vita (1960) et par Pier Paolo Pasolini dans Mamma Roma (1962) ${ }^{31}$. Quelques mois plus tôt, en décembre 2014, éclatait le scandale dit "Mafia capitale ", suite à l'enquête "Mondo di mezzo ». Tout au long des années précédentes, les faits et gestes de la pègre romaine alimentent une production romanesque foisonnante. Protagonistes d'une épopée nostalgique sans précédent, les criminels seront pratiquement «adoptés » par la ville, qui façonne et actualise son image au gré des effets de la transposition multimédia de Romanzo criminale, le polar noir vite devenu un long-métrage puis une série télévisée. Sorti en 2002 de la plume d'un magistrat, Giancarlo De Cataldo, ce roman épique ${ }^{32}$ est inspiré de l'histoire de la banda della Magliana, une holding politico-criminelle qui a dominé Rome entre les années 70 et les années 80, en synergie avec la Mafia, la Camorra et L'ndrangheta (les trois organisations mafieuses «traditionnelles ») et qui a également été impliquée dans les très nombreux mystères politiques auxquels l'Italie républicaine de l'époque a été confrontée. Romanzo Criminale sera suivi en 2013 d'un autre roman, Suburra, qui revisite et met à jour les mêmes événements ${ }^{33}$.

Revenons à Saint-Jean Bosco. Pendant qu'un corbillard tiré par six chevaux noirs approche de la basilique, un hélicoptère lance des pétales de rose sur la foule tandis qu'une fanfare joue les thèmes musicaux du Parrain. Cette fois-ci, on n'est pas sur le tournage de Suburra, même si la réalité ressemble étrangement à la fiction ${ }^{34}$. Il s'agit des funérailles de Vittorio Casamonica, chef du clan homonyme, le « roi de Rome» $d$ 'après les affiches qui en annoncent le décès.

21 Les médias, unanimes, considèrent que cette mise en scène est à la mesure de l'impact du Parrain sur l'imaginaire criminel. Des comparaisons sont faites avec d'autres cérémonies célèbres de l'histoire des organisations mafieuses. Plusieurs sites d'information partagent de vieilles images des actualités cinématographiques du 2 février 1962 consacrées aux funérailles de Lucky Luciano, qui ont eu lieu à Naples le 29 janvier 1962. Sur le web résonne la voix de stentor héritée des années de la propagande 
fasciste : "La cérémonie religieuse a lieu dans l'église de la Trinité. Puis le cortège funèbre se dirige vers le cimetière anglais. Le frère de Lucky Luciano suit le corbillard tiré par huit chevaux $»^{35}$.

Parallèlement, sur les réseaux sociaux, les utilisateurs cinéphiles affichent les scènes métaphysiques d'un cortège funèbre qui traverse les faubourgs d'une Palerme postmoderne et apocalyptique. Il s'agit d'une séquence tirée de L'Oncle de Brooklyn [Lo zio di Brooklyn] de Daniele Ciprì et Franco Maresco, tourné en 1995 ${ }^{36}$. Des membres de la famille Casamonica accordent des interviews, passent à la télé, ouvrent les portes de leurs maisons et de vieux albums de famille pour montrer leur visage naturel, humble et modeste. L'hebdomadaire Oggi obtient en exclusivité et publie deux photos des funérailles avec corbillard à chevaux du père (1967) et de la mère (1977) de Vittorio Casamonica $^{37}$. Ces photos sont de toute évidence une "démonstration de puissance, d'impunité et de mauvais goût ", et en même temps elle constituent « un [...] élément nouveau dans la stratégie de construction du consensus social [...] pour se renforcer et effacer l'image de "parents pauvres" de la pègre romaine ${ }^{38}$. Largement reprises par les médias en ligne, elles dépassent vite les frontières italiennes. Les mélodies de Nino Rota, l'ombre de Don Vito Corleone, la proximité géographique des studios prestigieux de Cinecittà, le souvenir de Fellini et de Pasolini, sont un patrimoine iconographique facile à investir sur le marché mondial de l'information.

La Direction nationale antimafia a effectivement constaté que "le rite funèbre spectaculaire et extravagant organisé par les représentants du clan historique des Casamonica [...] pour célébrer la mort du chef de famille, Vittorio, a ravivé l'intérêt de la presse, y compris à l'international $»^{39}$.

De son côté, le monde de l'information et de la communication frôle souvent le voyeurisme à l'égard des Casamonica ${ }^{40}$. A tel point que Vera Casamonica, fille de Vittorio, est invitée sur le plateau de Porta a porta, l'une des émissions phare de Rai Uno, où elle s'exclame: "Là-haut, il est heureux parce qu'on a fait les funérailles qu'il aimait. [...] Pour nous, c'était un roi. C'était un bon père, il ressemblait au bon Pape, c'était Wojtila ». La légitimité d'une telle intervention sur une chaîne du service public a été immédiatement questionnée : «Ce fut le triomphe de l'iconographie Casamonica, représentée à l'écran par des invités très sûrs de leur fait [...] madame [en français dans le texte, $n d t]$ Vera a parlé de la bonté de son papa, Vittorio, de son excellent sens des affaires [...]. Un affront au bon sens et à l'image de la Rai dans son ensemble $»^{41}$.

A rebours, allons maintenant du côté de Palerme, dans les années 80. Ce sont les années enflammées du «maxi-procès» contre Cosa Nostra (1986-1987), qui parviendra à détruire le mythe de l'invulnérabilité de la mafia et à rétablir la souveraineté de l'État. Les Italiens voient défiler dans la salle d'audience-bunker du tribunal de Palerme des centaines d'accusés filmés par les caméras de la Rai, grâce à « une impressionnante machine de production [...] capable d'enregistrer les audiences intégralement et en exclusivité, à la demande aussi du ministère de la Justice $»^{42}$. Parmi eux, une place de choix a été réservée à Michele Greco, le «Pape » de Cosa Nostra, confronté au «soldat mafieux " et témoin coopératif Salvatore Contorno, dans le but de décrédibiliser sa déposition mettant en cause un avocat de Palerme (Salvatore Chiaracane) qu'il accusait d'être associé à un clan ${ }^{43}$. Greco a développé un discours suivant deux axes destinés à converger: d'un côté, l'éthique mafieuse, de l'autre, le cinéma comme agent de l'histoire, capable de «structurer les comportements, promouvoir les passions, les choix collectifs $»^{44}$. Greco s'adresse en ces termes au juge Giordano: «La ruine de 
l'humanité, ce sont certains films. Les films violents, les films pornographiques, voilà la ruine de l'humanité. Car si, au lieu du Parrain, Contorno avait vu Moïse, il n'aurait pas calomnié l'avocat Chiaracane. Sûr et certain $»^{45}$.

L'ironie de l'histoire, c'est que le fils de Michele Greco, Giuseppe, « avait trouvé dans le cinéma son obscur objet du désir. Et en 1981, avec l'argent de son père, il avait pu produire un film qui, dès son titre, rappelle les comédies des années 70 : Crema, cioccolata e pa... prika $»^{46}$. Des années plus tard, sous le pseudonyme Giorgio Castellani, il réalise deux longs métrages: Vite perdute (1992), remake mafia-phile du film culte Ragazzi fuori de Marco Risi (1990) et I Grimaldi (1997), " portrait indirect de son père, joué par une sorte d'imitateur du Brando du Parrain ${ }^{47}$.

Dans la pénombre de son bureau, savamment créée par le directeur de la photographie Gordon Willis ${ }^{48}$, Don Vito Corleone a l'air d'un homme paisible, dont la richesse est fondée sur ses relations avec des journalistes, des politiciens, des juges, des showmen; un homme ne voulant surtout pas se lancer dans le trafic de drogue, un bon père de famille, un grand-père tendre, un parrain accueillant, bienveillant, capable de rendre la justice à ceux qui en sont privés, sans pour autant mépriser le rêve américain.

[Le Parrain], dont la production est d'abord contestée par Joe Colombo, un boss de Cosa Nostra [...], devient la parfaite autoreprésentation de la mafia, gardienne des valeurs anciennes, telles que la famille, la justice, l'honneur [...]. Un enquêteur nous a dit que dans ces années-là, dans toute maison perquisitionnée dans le cadre d'une enquête, le FBI trouvait presque toujours des cassettes VHS du film de Coppola ${ }^{49}$.

\section{Et voici un passage significatif du roman de Mario Puzo :}

Don Vito Corleone était un homme à qui tout le monde venait demander aide et assistance et qui jamais ne décevait ceux qui s'adressaient à lui. Jamais il ne faisait de vaines promesses ; jamais il ne cherchait à se dérober en alléguant qu'il existait dans le monde des puissances supérieures qui lui liaient les mains; il eût tenu cette excuse pour lâcheté. Il n'était pas nécessaire qu'il fût personnellement votre ami ; il importait peu que vous fussiez ou non en mesure de le payer de retour. Il n'avait qu'une exigence : que vous vous déclariez vous-même son ami. Dès lors, que son solliciteur fût obscur ou puissant, riche ou pauvre, Don Corleone partageait son souci [...] Quelle récompense en attendait-il ? Une amitié, l'honneur de s'entendre respectueusement appeler «Don » Corleone et quelquefois, plus affectueusement : padrino. Et peut-être aussi, mais seulement comme un gage de reconnaissance jamais comme un salaire, un modeste cadeau : un gallon de vin fait à la maison ou un panier de tamales spécialement préparés pour orner la table de la famille Corleone à la Noël. Par ces petits hommages, il était tacitement entendu que vous vous reconnaissiez comme l'obligé de Don Corleone, lequel pouvait à tout moment invoquer la dette que vous aviez contractée envers lui pour vous demander de lui rendre, en retour, quelque petit service ${ }^{50}$.

Ce n'est pas un hasard si Michele Greco, au cours d'un interrogatoire devant les juges, déclare : « La violence est étrangère à ma dignité. [...] Ma vie, c'est une vie rangée " $^{51}$.

31 Le storytelling des Casamonica et la stratégie défensive de Michele Greco démontrent que, pour être forte, « une organisation mafieuse doit [...] exploiter le patrimoine de la culture populaire afin d'obtenir le consensus des couches marginales de la société et renforcer l'image d'une mafia enracinée dans le passé, fondée sur des principes solides, peu sensibles à la modernisation, de manière à écarter le risque d'une mobilisation citoyenne adverse $»^{52}$. 


\section{Le home movie des Coppola}

du code des organisations criminelles, expose de fait les spectateurs et les chercheurs à une expérience culturelle très particulière. D'ailleurs, en 1972,

les associations représentatives de la communauté italo-américaine ont protesté et formé des piquets de grève devant les cinémas, contre les traîtres Coppola et Puzo. (Quelques années plus tard, le Order of Sons of Italy accusera Scorsese, De Niro et toute la troupe des Soprano de complicité dans un soi-disant « holocauste » italien). Le Parrain n'a certainement pas été le premier livre et film ayant comme protagonistes des criminels italiens, mais il a été le premier fait par des Italiens et centré sur les spécificités de la culture des communautés italiennes d'Amérique. [...] L'italianité avait-elle fait l'objet d'une stigmatisation négative et criminelle ? Et bien, il fallait donc revendiquer cette stigmatisation comme le signe d'une histoire de souffrance collective, transformée en épopée faisant partie du grand récit américain, toujours basé sur le système ou simplement sur le rêve de la mobilité sociale $^{53}$.

A distance de plusieurs décennies, en parcourant le press book du film ${ }^{54}$ donné aux journalistes italiens, lorsqu'on s'arrête sur la distribution technique et artistique de la trilogie, on a immédiatement le sentiment d'être confronté à une œuvre intégralement italo-américaine, où l'expérience de la migration du Vieux continent vers l'Amérique du Nord a joué un rôle essentiel tant au niveau de la création qu'au niveau de la production. En effet, Coppola « utilise son héritage culturel (méditerranéen) et, du grand réservoir de la tradition méridionale, puise une posture non manichéenne : le bien et le mal sont imbriqués et indissociables $\aleph^{55}$. Mario Puzo et Coppola sont italoaméricains, Al Pacino et Robert De Niro sont d'origine italienne, Nino Rota, l'auteur de la musique, est italien. La saga met en scène les campagnes et les villages de Sicile, le centre monumental de Palerme et Rome, à l'ombre du Vatican. Le Parrain n'est pas seulement un film tragique sur le pouvoir, la mafia, la trahison, l'Amérique des années $1970^{56}$ dans la vie d'une famille imaginaire, il est bel et bien une œuvre ethnoanthropologique très personnelle, fondée sur le vécu des émigrés, des ancêtres de Coppola, une sorte de home movie mélancolique, à faire pleurer dans les chaumières.

D'après John Yates, Coppola "présente son univers avec ce que Pauline Kael appelle une caméra ouverte : tout est montré, le détail est absolument convaincant; et c'est convaincant parce que le spectateur peut tout assimiler naturellement, sans commentaires du réalisateur ${ }^{57}$.

Lorsque le spectateur rencontre le soldat décoré Michael Corleone, dont l'héroïsme fait la fierté des siens (Clemenza, le bras droit de Don Vito, le dira explicitement), il le voit habillé de l'uniforme de l'armée américaine, accompagné d'une élégante beauté anglosaxonne, Kay Adams (un personnage qui n'assimilera jamais complètement l'esprit de la famille et qui sera toujours marginalisé ou plutôt, frappé d'une sorte d'exclusion «ethnique »). Écoutons à ce propos la femme de Coppola, Eleanor Jessie Nail : «Il semblerait que [Francis] y ait mis beaucoup de sa vie personnelle... de ses expériences en famille... [...] les gens me disent qu'il y a quelque chose en moi de Kay [...]. Diane m'a dit [...] qu'elle s'est inspirée de moi pour jouer son rôle. Je suis toujours un peu perplexe à l'idée qu'il y ait tant de ressemblance $»^{58}$.

Pour le rôle de Constanzia "Connie » Corleone, Coppola a choisi sa propre sœur, Talia Shire. Dans les scènes chorales du mariage de Connie avec Carlo Rizzi (Gianni Russo) ${ }^{59}$, 
parmi les invités au bal, la mère du réalisateur fait son apparition. Une partie des musiques populaires que l'on entend à plusieurs reprises et qui constituent une "métaphore de la fidélité à la culture d'origine ${ }^{60}$, ont été éditées par son père, Carmine Coppola, qui, tout comme sa femme, est aussi l'un des figurants lors du mariage sicilien de Michael et Apollonia.

37 Sofia Coppola, la fille du réalisateur, est présente dans les trois parties. Née en mai 1971, âgée d'à peine trois semaines, elle joue le bébé dans la scène du baptême de Michael Rizzi. Dans la deuxième partie, elle joue une petite fille qui voyage dans le paquebot qui emmène Vito-De Niro aux États-Unis. Dans la dernière, elle est destinée à une mort violente en tant que Mary Corleone, la fille de Michael. Talia Shire l'a dit franchement : «Le Parrain avait le goût et le parfum des souvenirs de notre famille ${ }^{61}$.

\section{Un mélodrame télévisé}

Aujourd'hui, à l'ère de la Complex $T V^{62}$, il est utile de poursuivre la réflexion à partir d'une définition du Parrain proposée en 1973 par John Gabree : somme toute, dit-il, ce film est conçu et réalisé comme un « un mélodrame télévisuel » ${ }^{63}$.

En effet, Le Parrain a connu un sort semblable à celui de tant de produits à succès : il est passé du grand au petit écran et s'est finalement imposé, par le bais de la sérialisation, en tant que visual novel. En novembre 1977 déjà, la National Broadcasting Company (NBC) avait réuni les deux premiers volets de la saga dans Mario Puzo's The Godfather: the Complete Novel for Television, diffusé en quatre soirées. L'épopée des Corleone s'est donc invitée chez les Américains avec neuf nouvelles scènes, précédemment coupées au montage: une heure de spectacle supplémentaire pour une intrigue chronologiquement différente, car les chapitres ayant pour protagoniste Robert De Niro ont été montrés au début. Manifestement, le modèle de référence étaient «les séries télévisées, qui font tourner la machine de la production en exploitant le succès de l'un de leurs films pilote $»^{64}$.

La sérialisation télévisuelle - surtout dans sa forme actuelle - reste une adaptation, mais il s'agit aussi d'une réélaboration susceptible de restituer au spectateur les rythmes puissants et impétueux des œuvres littéraires qui l'ont inspirée. "Les séries » en effet « ressemblent au roman d'apprentissage du XIXe siècle, qui accorde une grande place à tous les personnages, qui est choral et met en scène un grand nombre de conflits $»^{65}$.

41 Ce «regard a posteriori» permet de mieux interpréter la dynamique des transformations narratives qui ont investi Le Parrain - produit global, brand, écosystème narratif - et de la sérialité télévisuelle italienne contemporaine, qui a connu son apogée avec l'adaptation de trois œuvres littéraires appartenant au genre crime story: Romanzo criminale et Suburra, dont nous avons déjà parlé, et Gomorra de Roberto Saviano ${ }^{66}$.

Luca Bandirali et Enrico Terrone caractérisent ainsi cette solution narrative :

Le roman d'apprentissage en tant que lutte de succession est une structure récurrente dans les sagas criminelles, comme le montre le cas paradigmatique $d u$ Parrain [...]. Mais le roman d'apprentissage en tant que lutte de succession est aussi un thème crucial de la sérialité contemporaine. En ce sens, Gomorra - La Série est très proche de ses précurseurs cinématographiques, mais surtout des séries de son époque : Game of Thrones, [...] Alias, 24, Homeland, Mad Men ou encore Breaking Bad [...]. 
Pourquoi le conflit générationnel est si important dans les séries contemporaines? [...] D'abord, parce la série est un récit long qui permet de montrer le vieillissement des pères et le mûrissement des enfants avec une précision normalement impossible au cinéma. Ensuite, parce que la série traite d'une anomalie propre à la civilisation occidentale contemporaine: l'absence de renouvellement générationnel, la réticence des pères à céder la place à leurs enfants. Enfin, s'agissant de conflit des générations, la série parle aussi d'elle-même, de sa relation avec son propre père, le cinéma ${ }^{67}$. continue d'exister, une telle démarche narrative conduit à croire que cette série est plutôt un métadiscours sur la mafia, une œuvre de "méta-mafia " $»^{71}$ où les mafieux déprimés, accablés de crises de panique, entre deux séances chez le psy - déplorent l'époque contemporaine. Incapables de lire entre les lignes du présent ou d'imaginer un avenir, ils s'abandonnent au regret mélancolique du temps jadis et songent à un âge d'or qui n'est plus, à la bonne nourriture des repas d'antan. Grâce aux Soprano, nous avons compris que même la mafia peut être nostalgique d'elle-même.

\section{A table avec Don Vito : une offre que l'on pourra refuser}

A l'ère de l'homo dieteticus ${ }^{72}$, la dyade " mafia-Parrain »- entre épique et folklore, entre conscience et irresponsabilité - a connu une curieuse triangulation avec le secteur œnograstronomique, au point d'attirer l'attention de la Cour de Justice de l'Union européenne.

En mars 2018, le Tribunal de l'UE diffuse un communiqué de presse intitulé «La marque "La Mafia se sienta a la mesa" est contraire à l'ordre public », sous-titré : «L'Italie 
demande avec succès la nullité de l'enregistrement de cette marque en tant que marque de l'Union européenne $»^{73}$.

Que s'était-il passé ? En 2006, la société espagnole "La Honorable Hermandad ", à laquelle a succédé "La Mafia Franchises ", avait déposé auprès de l'office de l'Union européenne pour la propriété intellectuelle (EUIPO) une demande d'enregistrement de la marque "La mafia se sienta a la mesa ", une chaîne de restaurants italiens décorés en «style mafieux» et présents en Espagne depuis 2002. La marque, sur fond noir, était composée d'une rose rouge inclinée de la droite vers le centre pour s'approcher, sans la chevaucher, de l'inscription en grandes lettres "La Mafia », qui occupait l'espace au centre-gauche. Au dessous, sous une ligne de démarcation blanche, campait l'inscription « se sienta a la mesa » :

Les deux créateurs espagnols de ces restaurants, plus que par la culture sicilienne et la cuisine méditerranéenne, semblent avoir été influencés par la mafia et la culture mafieuse, à tel point que pour la décoration des locaux, ils se sont inspirés du film Le Parrain. Chaque petit détail de l'ameublement et de l'image de cette marque est conçu pour pousser à croire que la mafia et la culture culinaire italienne ne font qu'un $^{74}$.

En 2015, l'Italie a déposé une demande auprès de l'EUIPO visant à faire déclarer nulle cette marque, au motif qu'elle était contraire à l'ordre public et aux bonnes mœurs. Cette demande a été accueillie par l'EUIPO. En effet, l'EUIPO a considéré, d'une part, que la marque "La Mafia se sienta a la mesa» promouvait de façon manifeste l'organisation criminelle connue sous le nom de Mafia et, d'autre part, que l'ensemble des éléments verbaux de cette marque traduisait un message de convivialité et de banalisation de l'élément verbal « la mafia », déformant ainsi la gravité véhiculée par celui-ci ${ }^{75}$

Suite à cela, «Insatisfaite de la décision de l'EUIPO, "La Mafia Franchises" a saisi le Tribunal de l'Union européenne pour en demander l'annulation ", niant que la marque avait un " caractère offensant, car dans son intention la marque s'inspire des valeurs familiales et de convivialité véhiculées par la saga Le Parrain » ${ }^{76}$.

Mais le Tribunal de l'UE considère d'abord, que l'intention de La Mafia Franchises d'enregistrer la marque «La Mafia se sienta a la mesa » en vue d'évoquer la saga cinématographique Le Parrain, et non de choquer ou d'offenser, est sans incidence sur la perception négative de cette marque par le public. Il précise également que la réputation acquise par la marque de la société espagnole ainsi que son concept de restaurants à thème liés aux films de la saga Le Parrain sont dépourvus de pertinence aux fins d'apprécier si la marque est contraire à l'ordre public ${ }^{77}$.

Le Ministère italien des affaires étrangères diffuse à son tour un communiqué avec une déclaration du ministre Angelino Alfano:

L'image de notre Pays et des Italiens dans le monde est un patrimoine [...] à promouvoir et à protéger. [...] Cette entreprise, en utilisant le nom d'une des organisations criminelles les plus dangereuses qui existent, porte atteinte à l'image de l'Italie, notamment à la réputation extrêmement positive de la cuisine italienne, et surtout offense de manière inacceptable la sensibilité des victimes ${ }^{78}$.

Clairement, quels que soient les points de vue et les perspectives, la Mafia est toujours une question d'image. 


\section{Le Parrain, une allégorie de politique étrangère}

Il nous semble opportun de conclure par quelques remarques concernant les implications politiques du Parrain. Les brèves déclarations d'Henry Kissinger et de Barack Obama mises à part ${ }^{79}$, on peut évoquer un ouvrage fort efficace de deux politologues, John C. Hulsman et A. Wess Mitchell, The Godfather Doctrine: A Foreign Policy Parable (2011). Il s'agit d'un pamphlet qui propose une lecture métaphorique du scénario global de l'après-11 septembre. Les personnages de la trilogie de Coppola (notamment Don Vito, Tom, Sonny, Michael) sont décryptés du point de vue de la science politique. Ainsi Don Vito, désormais âgé, victime de l'embuscade des hommes $\mathrm{du}$ « turc » Sollozzo, serait « le symbole de la puissance américaine pendant la guerre froide. [...] Soudainement et violemment frappé par une force qu'il n'avait pas prévue et n'arrive pas à comprendre, comme ce fut le cas en Amérique le 11 septembre ", tandis que ses trois possibles héritiers seraient la représentation des «trois grands courants de la politique étrangère américaine $»^{80} \mathrm{du}$ début du XXIe siècle.

Tom Hagen, le fils adoptif-conseiller d'origine germano-irlandaise, promoteur raffiné d'accords et d'alliances, « est le produit d'une vision du monde juridico-diplomatique qui partage un certain nombre d'analogies philosophiques avec le liberal institutionalism ${ }^{81}$. Sa phrase préférée, en quelque sorte son identifiant politique : « Nous devrions leur parler ». Santino-Sonny, le fils aîné, l'héritier, est « l'archétype cinématographique du néoconservateur extrémiste $»^{82}$. D'après lui, les problèmes auxquels les Corleone doivent faire face après l'attaque contre le chef de famille peuvent être résolus par "une action militaire, une recette politique unique pour mener une guerre "juste" contre le reste du monde mafieux ingrat " $^{83}$. "Tire d'abord, tu réfléchiras ensuite", c'est sa devise. Et pour terminer, il y a le héros de guerre, le cadet, le moins expérimenté, le personnage qui, aux yeux de Coppola, incarne «un intellectuel conscient de sa propre tragédie ", capable en même temps d'élaborer « la stratégie qui préserve les Corleone du danger » auquel ils sont exposés dans ce monde multipolaire ${ }^{84}$. Michael le réaliste sait alterner « puissance douce et puissance dure [...] la carotte et le bâton ", utiliser tantôt l'esprit réparateur de Tom tantôt le désir de représailles de Sonny. Michael vit de tactique, en gardant à l'esprit l'objectif stratégique fondamental : « la survie et le bien-être de la famille » ${ }^{85}$.

D'après l'historien David W. Ellwood, il est des films qui « font l'histoire, d'autres qui la réécrivent et d'autres encore qui l'inventent de toutes pièces $»^{86}$. Trinitaire, la saga de Coppola semble véritablement incarner toutes ces possibilités. C'est pourquoi on peut supposer que l'évolution méta-discursive de cet extraordinaire outil de communication qu'est Le Parrain ne s'arrêtera pas de sitôt ${ }^{87}$.

\section{BIBLIOGRAPHY}

A.D. (2018) Romanzi nel tempo. come la letteratura racconta la storia, Roma-Bari, Laterza. 
AFI Catalog - American Film Institute. En ligne. URL : https://catalog.afi.com/Catalog/ moviedetails/54023.

Aprile, Marianna (2015), « Parlano i Casamonica: "Non siamo mafiosi, i funerali li abbiamo sempre fatti cosi” ", Oggi, 24 août 2015. En ligne. URL : http://www.oggi.it/attualita/notizie/ 2015/08/24/parlano-i-casamonica-non-siamo-mafiosi-i-funerali-li-abbiamo-sempre-fatti-cosiesclusivo/]

Annullamento marchio ristoranti « la mafia se sienta a la mesa », 15 mars 2018. https://www.esteri.it/ $\mathrm{mae} /$ it/sala_stampa/archivionotizie/comunicati/annullamento-marchio-ristoranti.html Armstrong, Jennifer Keishin (2019), « The Sopranos: A revolutionary show we'll talk about forever », BBC, 10 janvier 2019. En ligne. URL : http://www.bbc.com/culture/story/20190108-thesopranos-a-revolutionary-show-well-talk-about-forever

Bocca, Riccardo (2015), « Vespa, Casamonica e la tv della vergogna », L’Espresso, 9 septembre 2015. En ligne. URL : http://espresso.repubblica.it/attualita/2015/09/09/news/vespa-casamonica-e-latv-della-vergogna-1.228728

Bonini, Carlo - De Cataldo, Giancarlo (2016), Suburra. Rome, capitale de la mafia, Paris, Métélié (édition originale : Suburra, Torino, Einaudi, 2013).

Brunetta, Gian Piero (2003), Guida alla storia del cinema italiano. 1905-2003, Torino, Einaudi.

Caccia Riccardo - Gerosa Mario, éd. (2013), Maestri in serie. L'Abc dei telefilm d'autore, Alessandria, Falsopiano.

Cantone, Umberto (2019), « I cinquant'anni del Padrino. Così i cliché sulla mafia diventarono un brand », La Repubblica, 10 janvier 2019.

Careddu, Stefano (2018), «Il Padrino di Francis Ford Coppola », Cinefilia ritrovata, 30 juin 2018. En ligne. URL : https://www.cinefiliaritrovata.it/il-padrino-di-francis-ford-coppola/

Chirico, Danilo (2015), « L'impunità dei clan, quei funerali show dei Casamonica a Roma ». En ligne. URL : http://www.dasud.it/limpunita-dei-clan-quei-funerali-show-dei-casamonica-aroma/

Chirico, Danilo - Carta, Marco, éd. (2017), Under. Giovani Mafie Periferie, Roma, Giulio Perrone Editore.

Cimmarusti, Ivan (2019), « La Cassazione: il clan Casamonica di Roma è mafia », Il Sole 24 ore. En ligne. URL : https://www.ilsole24ore.com/art/notizie/2019-04-30/la-cassazione-clancasamonica-roma-e-mafia-184746.shtml?uuid=ABPtcBtB

Contaldo, Francesco - Fanelli, Franco (2017) Hollywood \& colossal. Nascita, splendori e morte della grande Hollywood, Roma, Alpes Italia.

Cortini, Letizia, éd. (2013), Le fonti audiovisive per la storia e la didattica, Archivio audiovisivo del movimento operaio e democratico, Arcidosso (Gr), Effigi.

Crespi, Alberto (2018) [1ère éd. 2016], Storia d'Italia in 15 film, Roma-Bari, Laterza.

Cusimano, Salvatore - Costa, Gian Mauro, éd. (2009) L'isola in onda. Storia della Rai in Sicilia dalla Liberazione ai nuovi orizzonti mediterranei, Roma, Rai Eri.

Deaglio, Enrico (2010), Il raccolto rosso 1982-2010. Cronaca di una guerra di mafia e delle sue tristissime conseguenze, Milano, Il Saggiatore.

De Cataldo, Giancarlo (2002), Romanzo criminale, Torino, Einaudi (Romanzo criminale. Roman criminel, traduit de l'italien par Catherine Siné et Serge Quadruppani, Paris, Métélie, 2006) 
De Cataldo, Giancarlo (2016), Come si racconta una storia nera, Roma, Rai Eri.

Dickie, John (2007), Cosa Nostra. L'histoire de la mafia sicilienne de 1860 à nos jours, Paris, BuchetChastel.

« È morto il regista Giuseppe Greco il figlio del “papa” della mafia», La Repubblica-Palermo (2011), 12 février 2011. En ligne. URL : https://palermo.repubblica.it/cronaca/2011/02/12/news/ e_morto_il_regista_giuseppe_greco_il_figlio_del_papa_della_mafia-12378758/.

Fossati, Mauro (2018), « Il mangiare "mafioso" che piace tanto agli spagnoli », Blog Mafie-La Repubblica, 27 août 2018. En ligne. URL : http://mafie.blogautore.repubblica.it/2018/08/27/2168/ Gabree, John (1973), Gangster. From Little Caesar to The Godfather, New York, Pyramid. Gambino, Sonia (2016), «Il Padrino nell'immagine turistica di Savoca / The Godfather in the touristic image of Savoca ", Il capitale culturale. Studies on the Value of Culturale Heritage. En ligne. URL : https://riviste.unimc.it/index.php/cap-cult/article/view/1425

Gardaphé, Fred (2003), « Re-inventing Sicily in Italian American Writing and Film », MELUS, Vol. 28, n. 3, Italian American Literature.

Grasso, Aldo (2019), «I Sopranos, la prima puntata 20 anni fa: un'epopea sulla depressione più che sulla mafia ", Corriere della Sera, 10 janvier 2019. En ligne. URL : https://www.corriere.it/ spettacoli/19_gennaio_10/i-sopranos-prima-puntata-20-anni-fa-un-epopea-depressione-piu-chemafia-2dc2bf1c-14df-11e9-bode-82ca1617bf76.shtml

Gratteri, Nicola - Nicaso, Antonio (2017), L'inganno della mafia. Quando i criminali diventano eroi, Roma, Rai Eri.

Guerra, Michele - Martin, Sara - Rimini, Stefania, éd. (2018), Universo Gomorra. Da libro a film, da film a serie, Milano-Udine, Mimesis.

Hulsman, John C. - Mitchell, A. Wess (2011), The Godfather Doctrine. A foreign Policy Parable, Macerata, Liberilibri.

Lodato, Saverio (2005), Venicinque anni di mafia. C'era una volta la lotta alla mafia, Milano, Rizzoli.

Lupo, Salvatore (2002), « La mafia americana: trapianto o ibridazione? », Meridiana, n. 43, pp. 15-48.

Marcucci, Federica (2018), « Un canto epico per la violenza e la malinconia. Il padrino di Francis Ford Coppola », Cinefilia ritrovata, 30 juin 2018. En ligne. URL : https://www.cinefiliaritrovata.it/ un-canto-epico-per-la-violenza-e-la-malinconia-il-padrino-di-francis-ford-coppola/

Marino, Giuseppe Carlo (2006), Storia della mafia, Roma, Newton Compton.

Meccia, Andrea (2014), Mediamafia. Cosa Nostra fra cinema e Tv, Trapani, Di Girolamo Editore.

Melorio, Simona - Ravveduto, Marcello, éd. (2015), Dialoghi sulle mafie, Soveria Mannelli, Rubettino.

Michetti, Massimiliano (2017), LOOP. Dal cinema al videogame... e ritorno, StreetLib.

Milizia, Giulia (2018), « "La Mafia se sienta a la mesa”, stereotipo che offende l'Italia e l'UE: il marchio è nullos ", Diritto e Giustizia, 15 mars 2018. En ligne. URL : http://

www.dirittoegiustizia.it/news/17/0000088408/

La_Mafia_se_sienta_a_la_mesa_stereotipo_che_offende_l_Italia_e_1_UE_il_marchio_e_nullo.html? $\operatorname{coc}=4$ 
Mittell, Jason (2015) Complex TV: The Poetics of Contemporary Television Storytelling, New York, NYU University Press.

Monda, Antonio (2008), « Tutti contro Il Padrino », La Repubblica, 28 septembre 2008.

Morreale, Emiliano (2007), « Mafia-movie all'italiana », Lo Straniero, n. 82.

Niola, Marino (2015), Homo dieteticus. Viaggio nelle tribù alimentari, Bolonga, Il Mulino.

Poniewozik, James (2015), « Streaming TV isn't Just a New Way to Watch. It's a New Genre », The New York Times, 16 décembre 2015. En ligne. URL : www.nytimes.com/2015/12/20/arts/ television/streaming-tv-isnt-just-a-new-way-to-watch-its-a-new-genre.html?_r=0 Puzo, Mario (1970), Le Parrain, traduit de l'américain par Jean Perrier, Paris, Éditions Robert Laffont.

Rizzo, Giuseppe (2013), « Oggi ho ucciso cento mafiosi », Rivista Studio, 25 novembre 2013. En ligne. URL : https://www.rivistastudio.com/oggi-ho-ucciso-cento-mafiosi/

Rosi, Francesco - Tornatore, Giuseppe (2014), Io lo chiamo cinematografo, Milano, Mondadori. Russo, Gianni (2019), Hollywood Godfather. My Life in the Movies and in the Mob, New York, St. Martin's Press.

Sansonna, Giuseppe (2016), Hollywood sul Tevere. Storie scellerate, Roma, Minimum Fax.

Santopietro, Tom (2012), The Godfather Effect. Changing Hollywood, America and me, New York, St. Martin's Press.

Shadoian, Jack (1980), Il cinema gangsteristico americano. Sogni e vicoli ciechi, Bari, Dedalo Libri.

Szigeti, Balázs (2009) « The dialect of Sin in Shakespeare's Macbeth and Francis Ford Coppola's The Godfather Trilogy », The AnaChronisT, 14.

Talese, Gay (2015), Ton père honoreras, traduit de l'anglais par Yves Malartic, Paris, Editions du Seuil-Editions du sous-sol (édition originale : Honor Thy Father, Cleveland, World Publishing Company, 1971).

Tribunal de l'Union Européenne (2018), Communiqué de presse n. 33/18, 15 mars 2018. En ligne. URL : https://curia.europa.eu/jcms/upload/docs/application/pdf/2018-03/cp180033it.pdf.

Tribunale di Palermo (1982), Ufficio Istruzione Processi Penali n. 2289/82 R.G.U.I., OrdinanzaSentenza emessa nel procedimento penale contro Abate Giovanni + 706, Volume n. 23. URL : https://www.csm.it/documents/21768/1957009/ Ord+maxiprocesso+volume+23+pagine+4607_4835.pdf/af684a68-e344-e50f-3c26-199c187d7e37 Werner, Jeff (1990), The Godfather Family: A Look inside, TV Movie - Film documentaire.

Yates, John (1975), « Godfather Saga: The Death of the Family », Journal of Popular Film, Volume 4, Issue 2, pp. 157-163.

Zagarrio, Vito (1980), Francis Ford Coppola, Firenze, La Nuova Italia.

\section{NOTES}

1. Etaient présents : Francis Ford Coppola, Al Pacino, James Caan, Robert Duvall, Diane Keaton, Talia Shire et Robert De Niro. La soirée était animée par le réalisateur et producteur Taylor Hackford, https://www.tribecafilm.com/filmguide/archive/godfather-the-godfather-partii-2017. 
2. Dans son analyse des modes de consommation des séries télévisées, James Poniewozik, décrit le binge watching comme suit: "Regarder une série en streaming, c'est comme lire un livre [...] mais aussi comme jouer à un jeu vidéo. Le binge watching est immersif et contrôlé par l'utilisateur. Il crée une dynamique que j'appelle "l'aspiration" : un sentiment narcotique d'abandon qui fait qu'on se laisse emporter dans un spectacle qui vous absorbe pendant des heures. Vous appuyez sur "Regardez le prochain épisode", c'est tellement simple. Et ça peut même engendrer des compétitions. Vos amis affichent leurs progrès, heure par heure, sur les médias sociaux. [...]. Chaque épisode devient un palier à atteindre " (Poniewozik 2015). Sauf précision contraire, les citations en langue étrangère sont livrées en version française par la traductrice de cet article.

3. Cantone 2019.

4. Lupo 2002, p. 24

5. Talese 2015, pp. 388-389.

6. Engagé sur un projet de film noir de coût moyen (2,5 millions de dollars), le réalisateur a pu obtenir un budget plus important (6 millions) grâce à l'intervention in extremis de la compagnie pétrolière Gulf \& Western (cf. http://www.cinematik.it/2015/03/il-film-del-giorno-in-tv-ilpadrino.)

7. Cf. AFI Catalog - American Film Institute, https://catalog.afi.com/Catalog/moviedetails/54023.

8. Shadoian 1980, pp. 406-408.

9. Cit. in Zagarrio 1980, p. 80.

10. Brunetta 2003, p. 306.

11. Contaldo et Fanelli 2017, p. 215.

12. Careddu 2018.

13. Umberto Santino, fondateur $\mathrm{du}$ « Centro siciliano di documentazione Giuseppe Impastato » de Palerme, a précisé : «Il n'y a pas de doute que Coppola est un grand réalisateur et Marlon Brando un grand acteur, et le film sera un chef-d'œuvre, mais il est explicitement apologétique, c'est un véritable hymne à la mafia en tant que tutrice des valeurs anciennes : famille, justice, honneur etc., dans une société comme l'Amérique contemporaine, métaphore de l'univers, en pleine crise des valeurs et des repères " (cit. in Meccia 2014, p. 16).

14. A.D. «I fantasmi del "Cabaret” ", La Stampa, 3 septembre 1972.

15. Cf. https://www.cinematografo.it/cinedatabase/film/lamico-del-padrino/20989/.

16. Notons au passage que ce même Saro Urzì (comédien de qualité, souvent présent dans les films de Pietro Germi) avait joué le rôle du père d'Apollonia dans Le Parrin de Coppola.

17. Yates 1975, p. 159.

18. Gambino 2016.

19. Gardaphé 2003, p. 60.

20. Cf. https://www.afi.com/100years/movies10.aspx

21. Rosi - Tornatore 2014, pp. 303-304.

22. Sansonna 2016, p. 8.

23. Szigeti 2009, pp. 25-26.

24. Luciano Leggio à l'état civil, il a été présenté au parquet en tant que «Liggio » lors de sa première comparution. Au fil du temps, on a utilisé les deux noms. Ce boss était aussi connu sous son surnom, «le Mouron rouge de Corleone » [La Primula rossa di Corleone], faisant référence au héros-justicier du feuilleton anglais du début du XXe siècle, The Scarlet Pimpernel de la baronne Orczy, dont on a tiré un film à succès et plusieurs séries télévisées.

25. Giuseppe Fava, journaliste et écrivain, a été assassiné par la mafia, à Catane, en 1984. Cf. http://www.fondazionefava.it/sito/i-siciliani/i-cento-padroni-di-palermo/

26. Marino 2006, p. 282.

27. Reportage diffusé dans l'émission Rotocalco televisivo [magazine télévisé], animée par Enzo Biagi. Cf. http://www.teche.rai.it/2016/08/enzo-biagi-rotocalco-televisivo-1962/

28. Dickie 2007, p. 358. 
29. Ibid.

30. Deaglio 2010, p. 144.

31. Cf. Crespi 2018, p. 241.

32. De Cataldo 2002. De ce roman sont tirés le film Romanzo criminale de Michele Placido (2005) et la série télévisée Romanzo criminale (2008-2010, 2 saisons, Sky).

33. Bonini - De Cataldo 2013. De ce roman sont tirés le film Suburra de Stefano Sollima (2015) et la série télévisée Suburra (2017-2019, 2 saisons, Netflix).

34. Voici la description, dans Suburra, de l'enterrement de Cesare Adami, version fictive de Vittorio Casamonica : "Tirée par un quadrige d'étalons bais Haflinger qu'on avait fait venir du Tyrol, la chose en mouvement ressemblait au carrosse de Cendrillon. Mais c'était le corbillard de Cesare Adami [...]. Les bêtes, bardées de panaches noirs, avaient des parures en cuivre [...] Placé sur un immense coussin de roses rouges et blanches, le cercueil d'acajou occupait toute la longueur du carrosse laqué de noir [...]. Le trafic avait été bloqué par des patrouilles zélées de la police municipale, et la foule qui gonflait à son passage s'agrégea spontanément derrière le cercueil. Décidée à être là, mais surtout tenant à se faire voir. [...] Les boutiques avaient baissé leur rideau de fer. Le lieutenant Gaudino était fasciné. - Des funérailles comme celles-là, je n'en avais vu qu'à Forcella et dans les Quartiers Espagnols. La camorra a fait école », Bonini - De Cataldo 2016, pp. 316-317.

35. https://www.youtube.com/watch?v=R9AKAjJ-Z9Y

36. https://www.youtube.com/watch?v=t7o43plAA1w

37. Aprile 2015.

38. Chirico 2015.

39. Cf. Relazione annuale sulle attività svolte dal Procuratore nazionale e dalla Direzione nazionale antimafia e antiterrorismo, nonché sulle dinamiche e strategie della criminalità organizzata di tipo mafioso, février 2016, https://www.publicpolicy.it/wp-content/uploads/2016/03/RelazioneFranco-Roberti-Dna.pdf, p. 907.

40. "Une de leurs spécificités est que presque tous les mariages ont lieu au sein du clan, ce qui crée des liens de parenté qui unissent, du côté maternel ou du côté paternel, quasiment tous les foyers ; ce qui rend par ailleurs difficile l'identification des individus car ils portent souvent les mêmes prénoms ", Ibidem.

41. Bocca 2015.

42. S. Cusimano, La Rai al tempo dell'emergenza. Venticinque anni in prima linea, in Cusimano - Costa 2009, p. 194.

43. Tribunale di Palermo 1982

44. G. De Luna, Le nuove frontiere della storia. Il cinema come documento storico, in Cortini 2013, p. 40.

45. Blu Notte - La Mattanza, 25 juin 2003, https://www.raiplay.it/video-ssi.html?/video/2018/02/ Blu-notte---La-mattanza-bd900965-5d5c-4d8b-9bbd-d8617882efee-ssi.html

46. « È morto il regista Giuseppe Greco » 2011.

47. Morreale 2007, p. 51.

48. En 1970, au New York Film Festival, Coppola assiste à une projection du Conformiste de Bernardo Bertolucci, dont le directeur de la photographie avait été Vittorio Storaro. Déjà aux prises avec le scénario du Parrain, Coppola dit à Gordon Willis : " Je veux une photo comme cellelà !», cf. Crespi 2018, pp. 266-267.

49. Gratteri et Nicaso 2017, pp. 72-73.

50. Puzo 1970, p. 16.

51. Lodato 2005, p. 194.

52. M. Ravveduto, " "Il Male è tra noi". L'immaginario collettivo della camorra », in Melorio Ravveduto 2015, pp. 11-12.

53. S. Lupo, «La mafia americana. A partire da Il Padrino di Mario Puzo ", in A.D. 2018, pp. 195-196. 
54. Disponible à la consultation près la bibliothèque Luigi Chiarini du "Centro sperimentale di cinematografia" de Rome.

55. Zagarrio 1980, p. 82.

56. Présent à la première projection du film, le secrétaire d'état Henri Kissinger remarque : «C'est un film qui parle à tout le monde : ce n'est pas très différent de ce que je vois tous les jours à Washington » (Monda 2008). En 2016, le président Barack Obama dit : «Le Parrain II est peutêtre mon film préféré ", https://www.cnsnews.com/news/article/cnsnewscom-staff/obamagodfather-ii-maybe-my-favorite-movie

57. Yates 1975 , p. 158

58. Werner 1990.

59. Cf. Russo 2019, où le comédien raconte ses rapports avec des mafieux notoires et des célébrités du star system.

60. Marcucci 2018.

61. Werner, 1990.

62. Cf. Mittell 2015.

63. Gabree 1973 p. 122.

64. Zagarrio 1980, p. 93.

65. De Cataldo 2016, p. 61.

66. Du roman de Roberto Saviano, sorti en Italie en 2006, on a tiré le film Gomorra réalisé par Matteo Garrone (2008) et la série du même nom, en 4 saisons (2014, 2016, 2017 et 2019).

67. L. Bandirali - E. Terrone, Tele-Machia, in Guerra - Martin - Rimini 2018, pp. 24-25.

68. S. Martin, The Sopranos, alla ricerca di un cambiamento in-compiuto. Viaggio tra gli eroi archetipi di Francis Ford Coppola e quelli moderni di Martin Scorsese, in Caccia - Gerosa, 2013, p. 120.

69. Santopietro 2012, pp. 272-273. Cf. aussi https://www.youtube.com/watch?v=-EaR5x3Mh6M

70. Armstrong 2019.

71. Grasso 2019.

72. Cfr. Niola 2015.

73. Tribunal de l'Union européenne 2018.

74. Fossati 2018.

75. Tribunal de l'Union européenne 2018.

76.

http://www.dirittoegiustizia.it/news/17/0000088408/

La_Mafia_se_sienta_a_la_mesa_stereotipo_che_offende_l_Italia_e_1_UE_il_marchio_e_nullo.html? $\operatorname{coc}=4$

77. Tribunal de l'Union européenne 2018.

78. Annullamento marchio ristoranti 2018 (publié sur le site du Ministère italien des affaires étrangères).

79. Cf. note 53 .

80. Hulsman et Mitchell 2011, p. 26.

81. Id., p. 27.

82. Id., p. 33.

83. Id., p 32.

84. Id., p. 37.

85. Id., p. 38.

86. D.W. Ellwood, Cinema e didattica della storia oggi: una breve riflessione e un esempio, in Cortini 2013, p. 45.

87. L'univers des jeux vidéos a d'ailleurs offert de nouvelles opportunités à la fois esthétiques et structurelles à cette histoire. Voici quelques titres : Mafia, Mafia II, Il Padrino, MafiaShoot, Scarface, Mafia Wars II. Cf. notamment G. Rizzo 2013 ; Z. Gaiaschi et S. Troglio, Realtà digitali. La mafia è solo un gioco, in Chirico - Carta 2017, pp. 239-250; Michetti 2017. 


\section{ABSTRACTS}

Libro, film, visual novel, videogioco, allegoria politica: la saga della famiglia Corleone è tutto questo nell'immaginario collettivo mondiale da oltre mezzo secolo. Questo articolo torna sul percorso de Il padrino [The Godfather], il romanzo di Mario Puzo, e, esaminandone le trasformazioni mediatiche, ne valuta la capacità di tratteggiare, nella realtà postmoderna, un nuovo immaginario dell'universo mafioso, tanto all'interno quanto all'esterno delle associazioni criminali.

Livre, film, visual novel, jeu vidéo, allégorie politique : voilà ce qu'est la saga de la famille Corleone dans l'imaginaire collectif mondial depuis plus d'un demi-siècle. Cet article retrace le parcours du roman de Mario Puzo Le Parrain [The Godfather] et analyse ses transformations médiatiques ainsi que sa capacité à dessiner, dans la réalité postmoderne, un nouvel imaginaire du monde mafieux, à l'extérieur comme à l'intérieur des associations criminelles.

\section{AUTHORS}

\section{ANDREA MECCIA}

Andrea Meccia, journaliste, est directeur de la revue Questione Giustizia. Membre de l'association anti-mafia daSud, il est l'auteur de MediaMafia. Cosa nostra tra cinema e TV (2015) ainsi que coauteur de Strozzateci tutti (2010), une anthologie sur les mafias italiennes (Aliberti 2010), et de Novantadue. L'anno che cambiò l'Italia (ouvrage dirigé par Marcello Ravveduto, Castelvecchi 2012). Il a participé aux documentaires Ezio Tarantelli. La forza delle idee (Monica Repetto e Luca Tarantelli, Derivafilm 2010) et Voi siete qui (Francesco Matera, 2011, présenté au Festival de Venise 2011), en tant que responsable de production. Il a travaillé au service de presse de l'association Magistratura Democratica. 